\title{
Location Based Video Searching
}

\author{
Prof Aparna S Kalaskar, Kaustubh Karanjkar², Harish Gwalani ${ }^{3}$, Naman Jain ${ }^{4}$, Shridhar Pawar ${ }^{5}$ \\ Assistant Professor, Department of Computer Engineering, Sinhgad College of Engineering, Pune, India ${ }^{1}$ \\ Student, Department of Computer Engineering, Sinhgad College of Engineering, Pune, India ${ }^{2}$
}

\begin{abstract}
Location based Video Searching (LBVS) systems have been shown as valuable tools for providing appropriate recommendations to users. In the last decade, the amount of customers, services and online information has grown rapidly. Moreover, most of existing service recommender systems present the same ratings and rankings of services to different users without considering diverse users' preferences, and therefore fails to meet users' personalized requirements. We will develop Smart (LBVS), to address the above challenges. It aims at presenting a personalized service recommendation list and recommending the most appropriate services to the users effectively. Specifically, keywords are used to indicate users' preferences, and a user-based Collaborative and Demographic Filtering algorithm is adopted to generate appropriate recommendations. Extensive experiments are conducted on real-world data sets, and results demonstrate that Smart Recommendation System significantly improves the accuracy and scalability of service recommender systems over existing approaches. Geo-location is a feature that is presented individually to users. The users can share location and add geo-located data to content. Adding geographical identification metadata to an information resource attach value to the content by making it more searchable.
\end{abstract}

Keywords: Location based Video Searching, customer reviews, Geo-location, metadata.

\section{INTRODUCTION}

Geotagging (also written as GeoTagging) is the process of adding geographical identification metadata to various media such as a geotagged photograph or video, websites, SMS messages, QR Codes[1] or RSS feeds and is a form of geospatial metadata. This data usually consists of
latitude and longitude coordinates, though they can also include altitude, bearing, distance, accuracy data, and place names, and perhaps a time stamp. Geotagging can help users find a wide variety of location-specific information from a device. For instance, someone can find images taken near a given location by entering latitude and longitude coordinates into a suitable image search engine. Geotagging-enabled information services can also potentially be used to find location-based news, websites, or other resources.[2] Geotagging can tell users the location of the content of a given picture or other media or the point of view, and conversely on some media platforms show media relevant to a given location.

Automatically generated tags and geotags hold great promise to improve access to video collections and online communities. We overview three tasks offered in the MediaEval 2010 benchmarking initiative, for each, describing its use scenario, definition and the data set released. For each task, a reference algorithm is presented that was used within MediaEval 2010 and comments are included on lessons learned. The Tagging Task, Professional involves automatically matching episodes in a collection of Dutch television with subject labels drawn from the keyword thesaurus used by the archive staff. The Tagging Task, Wild Wild Web involves automatically predicting the tags that are assigned by users to their online videos. Finally, the Placing Task requires automatically assigning geo-coordinates to videos. The specification of each task admits the use of the full range of available information including user-generated metadata, speech recognition transcripts, audio, and visual features. This will be a work of geo-tagging video content with metadata attached and clipping it accordingly. Also this is a problem that allows restaurant/chefs/tastemakers to showcase their products.

\section{RELATED WORK}

This section provides a brief overview of relevant literature on automatic tagging, including supporting information on related work in speech indexing, geotagging and benchmarking initiatives related to multimedia retrieval and indexing.

\subsection{Tagging and Subject Label Generation}

The purpose of assigning labels to content is to achieve a representation that encodes a higher level of semantic abstraction. Within multimedia information retrieval systems, indexing features provide high-level semantic generalizations concerning individual items. Automatic approaches to label or tag prediction generally follow one of the two approaches, distinguished by [11]. The first approach involves extraction. Here, appropriate labels for items are chosen from among the words or phrases already associated with item content or metadata. Under this approach, the identity of the labels to be assigned need not be known in advance, a task we refer to as 'open-set tagging'. The second approach involves assignment. Here, 
labels from a fixed set of labels are assigned to items, a task we refer to as 'closed-set tagging'.

\subsection{Automatic Geotagging of Multimedia}

Previous work that has been carried out in the area of automatic geotagging of multimedia has focused on images, usually from Flickr. User-contributed tags have a strong location component, as brought out by [33], who reported that over $13 \%$ of Flickr image tags could be classified as locations using Wordnet. In [29], the geolocations associated with specific Flickr tags are predicted using spatial distributions of tag use. A tag which is strongly concentrated in a specific location has a semantic relationship with that location. Understanding that a tag (or a term) is highly correlated with a place is the key to understand whether a user has a geographic intent in mind when tagging an image or searching for images on the Web. User-contributed tags are exploited for geotagging by [32], who use tag distributions associated with locations represented as grid cells on a map of the Earth is used to infer the geographic locations of Flickr images. Tag prediction methods exploiting the visually depicted content of images include [14], which uses visual features and a nearest-neighbor classification method to geotag Flickr images. The data set is, however, limited to a subset of Flickr images tagged with at least one name of a country, continent, densely populated city or popular tourist site and not tagged with specific non-geographic tags such as birthday or concert. Visual, textual and temporal features are combined by [7], which investigates the classification of images within specific cities. For 100 cities, the top ten landmarks in that city are identified and 10-way classification of photos geotagged around these landmarks is performed. Other closely related work addresses geotagging of non-multimedia objects, for instance finding the geographical focus of Web pages [2] or short Twitter messages [4].

\section{III.PROPOSED SYSTEM ARCHITECTURE}

It aims at presenting a personalized service recommendation list and recommending the most appropriate services to the users effectively. Specifically, keywords are used to indicate users' preferences, and a user-based. To improve its scalability and efficiency in big data environment

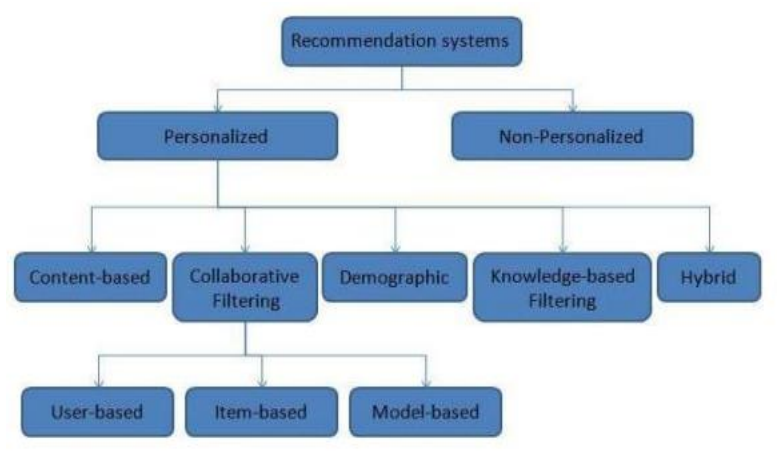

There are several approaches to opinion mining in the current research. Opinion mining is the area of research that attempts to create automatic systems to determine human opinion, with the aim of extracting attributes and components of the object that have been commented upon in each document. It is meant to determine from text written in natural language whether the comments reflect positive, negative or neutral opinions [4]. It starts with sentiments bearing lexical items, known as "Sentiment Classification," and investigates ways to classify each review document as positive, negative or neutral.

Some researchers do it at the document level, whereas others work at the sentence level. The methods are essentially based on two types of approaches: 1) corpusbased approaches that find co-occurring patterns of words to determine the sentiments of words or phrases and 2) dictionary-based approaches using synonyms and antonyms in WordNet to determine word sentiments based on a set of seed opinion words.

\subsubsection{Opinion Mining and Customer Reviews}

In their research, Minqing and Bing reported on their process of mining and summarizing customer reviews in three main steps. First, they mined product features that had been commented on by customers, utilizing both data mining and natural language processing techniques to perform this task. Second, Minqing and Bing identified opinion sentences in each review and decided whether each opinion sentence was positive or negative. To determine the opinion orientation of each sentence, three subtasks were performed. First, a set of adjective words was identified using a natural language processing method. Second, the semantic orientation of each opinion word was determined. Finally, the opinion orientation of each sentence was assessed [3].

\subsubsection{Opinion Mining in Hotel Customer Reviews}

In December 2012, a study applied opinion mining to data from travel review sites and examined how the results of sentiment analysis of textual reviews can be visualized using Google Maps. The researchers used SentiWordNet in their application then found and extracted six features from Booking.com and Trip Advisor: "breakfast," "staff," "service," "clean," "location" and "Internet." This led to the provision of "good" and "bad" hotels as geographical areas, based on hotel reviews [13].

In 2011, system architecture for the opinion-mining framework was proposed, which consisted of two main modules: language resource construction and opinion mining modules. In their experiment, Kasper and Vela, collected hotel reviews from the Agoda website to generate a feature-based summary and comparison between hotels, based on customer reviews in Thailand. The system, called HotelOpinion, asks users to identify the city where they wish to stay. The system then displays all of the hotels in that specific city. Users can then select the name of the hotel where they prefer to stay. Based upon 
the user's selection, the system classifies and displays reviews for that particular hotel, based upon the following features determined earlier: "service," "hotel condition," "location," "food," "room condition," "facilities" and "price" [14].

Kasper and Vela presented a web-based opinion-mining system for hotel reviews. This system is capable of detecting and retrieving reviews on the web. The target users for this system are hoteliers who want to obtain actual overviews and summaries of textual comments about their hotel(s) on the web. With the acquisition system, data can be retrieved from the web and then stored in the hotel database and transferred to the analysis system, which is handled by a web crawler. A content extraction module is applied to the retrieved target page to extract the relevant textual content of the review and other information about the reviewer.

The analysis system checks the language to filter out reviews in other languages (this system is only available in German) and it is stored in the review database. Then the review text is segmented into sentences. Next the statistical polarity classifier divides the segments into subjects. The IE system is designed to find results regarding the following matters: the topic of the review segment, the dimension of evaluation, the dimension value and the polarity of the evaluation. After that, moving to finer-grained analysis of the polarity and the topic of the review, the linguistic information extraction is performed. Finally, the results and segments are stored in the results database [15].

\section{IV.ALGORITHM}

\section{Location Search Algorithm}

The search algorithm takes in a latitude, longitude and additional tags like "Italian", "North Indian" etc. which are used as additional filters.

1) It maps the latitude and longitude to the spatial zone to which it belongs to, and returns a list of restaurants in that zone.

2) It then looks for restaurants in adjacent spatial zones, and returns a list of restaurants in each of the nearby zone. If step one and step two return restaurants whose count is more than a defined critical count, the algorithm stops else it continues.

3) If step one and step two return no results, the algorithm looks for all the restaurants in the same city.

4) If even step three returns an empty result, the algorithm returns all the restaurants in the database limited to a defined count and ordered by a defined property.

Based on this search algorithm a Rails-Angular application called 'Chennai Foodie' was built, which enabled users to locate restaurants near their current location.

The application uses the phone's location via the HTML5 geo-location API and fetches the restaurants in and around the user. The user can additionally specify Cuisines (tags) to filter his/her result further.

As an enhancement, a free text search algorithm was also implemented which responds to queries like "Find me an Italian restaurant in Adyar" or "Get me all the restaurants near Guindy".

\section{Free text search Algorithm}

The text query is split into words. The algorithm loops through each word and compares it to a set of locality names, and cuisines name and compute a SQL query.Example: "Find me an Italian restaurant in Adyar" would result in a query like $->$ "select * from restaurants where cuisines in ['Adyar'] and locality in ['Guindy']"

It's to be noted that the entire system was built and architected using the TDD philosophy and is completely spec covered.

\section{CONCLUSION}

From the consideration of all the above points we conclude that, Location based Video Searching have been shown as valuable tools for providing appropriate recommendations to users. Consequently, traditional service recommender systems often suffer from scalability and inefficiency problems when processing or analyzing such large-scale data.

Moreover, most of existing service recommender systems present the same ratings and rankings of services to different users without considering diverse users' preferences, and therefore fails to meet users' personalized requirements. Opinion mining has become an interesting research area due to the availability of a huge amount of user-generated content, such as booking websites, forums and blogs.

Automatically generated tags and geotags can supplement user-contributed annotations and contribute to improving access to content in video collections and communities. We presented three tasks involving tagging and geotagging that were run in MediaEval 2010 along with descriptions. The major goal of marketing is to understand the consumer and to influence buying behavior, which is achieved by providing the final piece of work: the web-based user interface that enables users to select their preferred category, country and destination city, and then find the hotel that best matches their preferences.

\section{ACKNOWLEDGMENT}

We would like to express our sincere gratitude towards our guide Prof. A. S. Kalaskar for her invaluable guidance and supervision that helped us in our research. She has always encouraged us to explore new concepts and pursue newer research problems. I credit our project contribution to her. Collectively, we would also like to thank our 
project committee members Prof. G. D. Chavan and

Prof. S. C. Suryavanshi for their time, suggestions, and for graciously agreeing to be on our committee, and always making themselves available. We cannot thank them enough.

\section{REFERENCES}

[1] Automatic Tagging and Geotagging in Video Collections and Communities. Martha Larson Delft University of Technology Delft, the Netherlands m.a.larson@tudelft.nl Mohammad Soleymani University of Geneva Geneva, Switzerland mohammad.soleymani @unige.ch Pavel Serdyukov Yandex Moscow, Russia pavser@yandex-team.ru

[2] E. Amitay, N. Har'El, R. Sivan, and A. Soffer. Web-a-where: Geotagging web content. In SIGIR '04, pages 273-280, 2004.

[3] K. Chandramouli, T. Kliegr, T. Piatrik, and E. Izquierdo. QMUL @ MediaEval 2010 Tagging Task: Semantic query expansion for predicting user tags. In MediaEval '10 Working Notes, 2010.

[4] F. Chang, J. Dean, S. Ghemawat, and W.C. Hsieh, "Bigtable: A Distributed Storage System for Structured Data," ACM Trans. Computer Systems, vol. 26, no. 2, article 4, 2008.

[5] W. Dou, X. Zhang, J. Liu, and J. Chen, "HireSome-II: Towards Privacy-Aware Cross-Cloud Service Composition for Big Data Applications," IEEE Trans. Parallel and Distributed Systems, 2013. 